\title{
Prevención del cáncer de piel
}

BOLETÍN

Vol. 6

No 1

Elaborado por: Ana Córdoba Bastos-Interna Universitaria Revisado por: Dra. Wendy Montoya Vargas, Farmacéutica

\section{Tipos de radiación solar}

El espectro de radiación solar cubre regiones entre los 200 y $400 \mathrm{~nm}$ y es responsable de daño en la piel. Este espectro se puede dividir en:

UVC (100-280 nm): la radiación es filtrada por la capa de ozono por lo que no causa daño a superficies ${ }^{1}$.

UVB (280- $315 \mathrm{~nm}$ ): penetra las capas más externas de la piel, causando daños en el ADN y quemaduras solares ${ }^{1}$.

UVA (315-400 nm): alcanza las capas más profundas de la piel, causando fotocarcinogénesis y fotoenvejecimiento ${ }^{1}$.

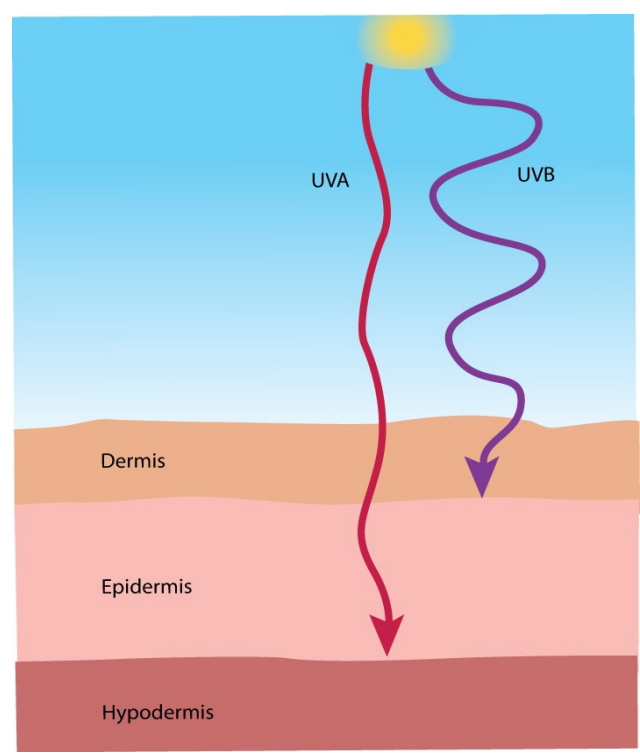

Los rayos UVA y UVB son clínicamente significativos porque ambos se han asociado con aumento del riesgo de cáncer de piel, fotoenvejecimiento y la inmunosupresión ${ }^{1,2}$.

\section{Bloqueadores solares}

Son productos diseñados para bloquear la radiación solar y proteger a las células de la piel contra los efectos nocivos de la radiación UV tales como las quemaduras solares y el cáncer de piel ${ }^{3}$.

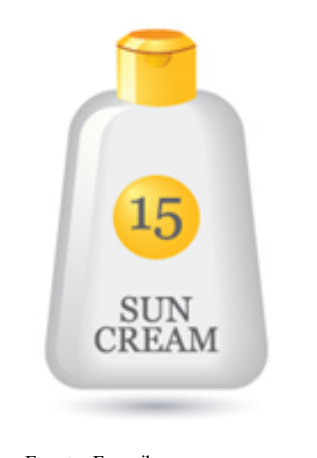

Fuente: Freepik
Los bloqueadores solares contienen sustancias atenuadoras conocidas como filtros solares y estos son los que tienen la capacidad de evadir la radiación incidente por distintos mecanismos $^{2,3}$.

Se debe de evaluar la seguridad y efectividad de los ingredientes activos, pues cada uno tiene capacidad protectora diferente. La FDA ha autorizado el uso, únicamente de 17 ingredientes y la concentración máxima a la que pueden ser formulados ${ }^{4}$. 


\section{Componentes de los bloqueadores solares}

Los bloqueadores solares pueden contener tanto ingredientes orgánicos, como inorgánicos:

Ingredientes inorgánicos: absorben una parte de la radiación que penetra la piel y además, provocan reflexión y dispersión de la radiación UV. Ejemplo: dióxido de titanio y óxido de Zinc ${ }^{1,5}$.

El mecanismo de atenuación de la radiación de los ingredientes inorgánicos se considera más efec- tivo que el de los ingredientes orgánicos y pueden ser más útiles en pacientes con piel sensible o enfermedades de irritación en la piel $^{5}$.

Ingredientes orgánicos: sólo absorben la radiación UV, de manera que son susceptibles a la fotodegradación y pueden llegar a generar radicales libres. Ejemplo: oxibenzona, p-aminobenzoico, avobenzona, ensulizole, octinoxa- to, octisalate ${ }^{1,5}$.

Los componentes orgánicos son más abundantes que los inorgánicos y brindan flexibilidad, resistencia al agua y una buena sensación al tacto 5 .

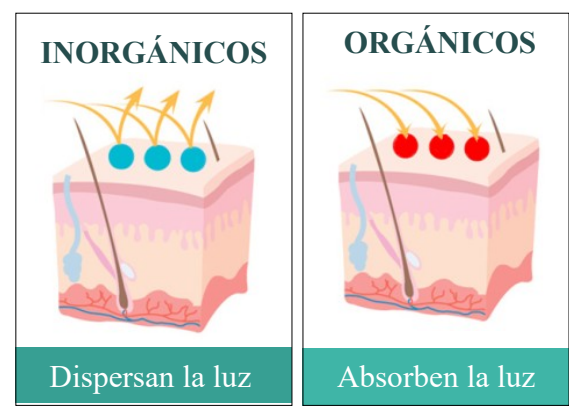

\section{Factor de Protección Solar (FPS)}

En muchos casos, es el único indicador de eficacia de los bloqueadores

El FPS es una relación entre el tiempo de exposición a la radiación UV que causa eritema en la piel con el uso de bloqueador solar entre el tiempo en que se obtiene la misma respuesta en la piel expuesta sin protector solar².

Ejemplo: Utilizar un FPS de 15 significa que el tiempo sin obtener una quemadura solar se extiende 15 veces más del tiempo usual en obtenerla sin protección solar.

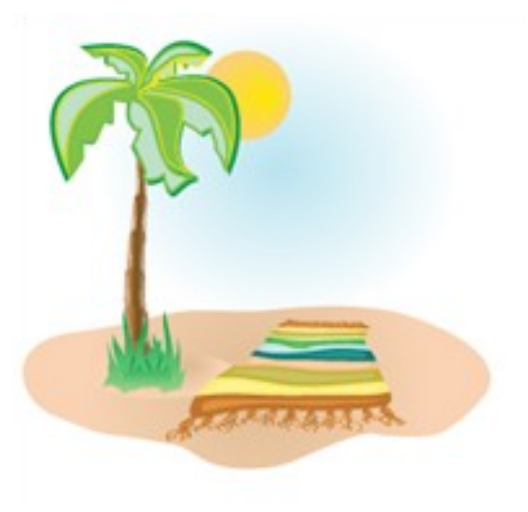

Fuente: Freepik
Sin embargo, es importante tener en cuenta, que la duplicación del FPS no implica que se duplique la protección UV, ya que por ejemplo, un FPS 15 bloquea el $94 \%$ de los rayos UVB, mientras que un FPS 30 bloquea aproximadamente el $97 \%$ de los rayos UVB. De hecho para los FPS mayores a 30 , el bloqueo es muy cercano a $100 \%{ }^{2}$.

Por ello la FDA propuso categorizar los niveles de protección en: baja ( FPS 8-15), media (FPS 15-30), alta (FPS 30-50) y la más alta (FPS $>50$ ). Se recomienda el uso de bloqueadores de al menos un FPS de $15^{2,6}$. 


\section{Medición de eficacia-Controversias}

El FPS mide la respuesta de la piel a la radiación $\mathrm{UVB}$, sin embargo, la radiación UVA tiene 1000 veces menos probabilidades de causar eritema en comparación con la exposición UVB por lo que se ignoran los efectos de la radiación UVA $^{2}$.
Según estudios, los FPS más altos ofrecen menor protección contra la radiación $\mathrm{UVA}^{2}$.

Adicionalmente, es importante conocer que el FPS no proporciona información acerca de la capacidad de los bloqueadores solares para soportar las condiciones acuosas como: el sudor y la natación ${ }^{2}$.

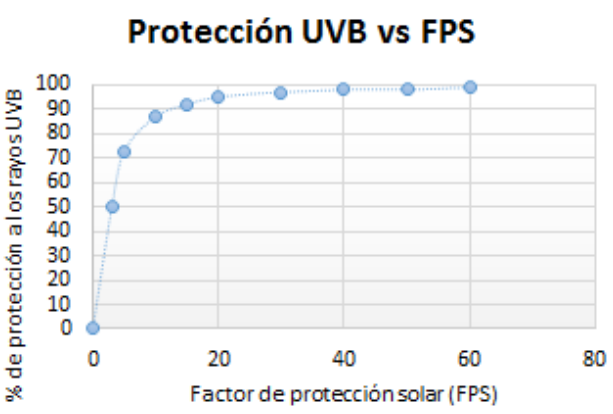

El FPS no proporciona información acerca de la resistencia al agua

\section{Alergenicidad}

Hay que tener precaución con este aspecto, principalmente en la población pediátrica

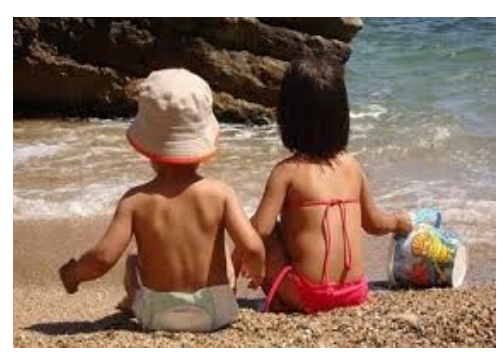

ya que son más susceptibles a desarrollar alergias. Los productos que indican estar "libre de PABA" son potencialmente menos alergénicos ${ }^{2}$.

Adicional a ello, la piel de los niños es más sensible a la radiación $\mathrm{UV}^{6}$ :

$\Rightarrow \quad$ La exposición al sol aumenta el riesgo de melanoma y de otros cánceres en el futuro.

$\Rightarrow \quad$ Los niños pasan más tiempo al aire libre, por lo que se exponen más a la radiación solar. 


\section{Recomendaciones para el uso adecuado del bloqueador solar}

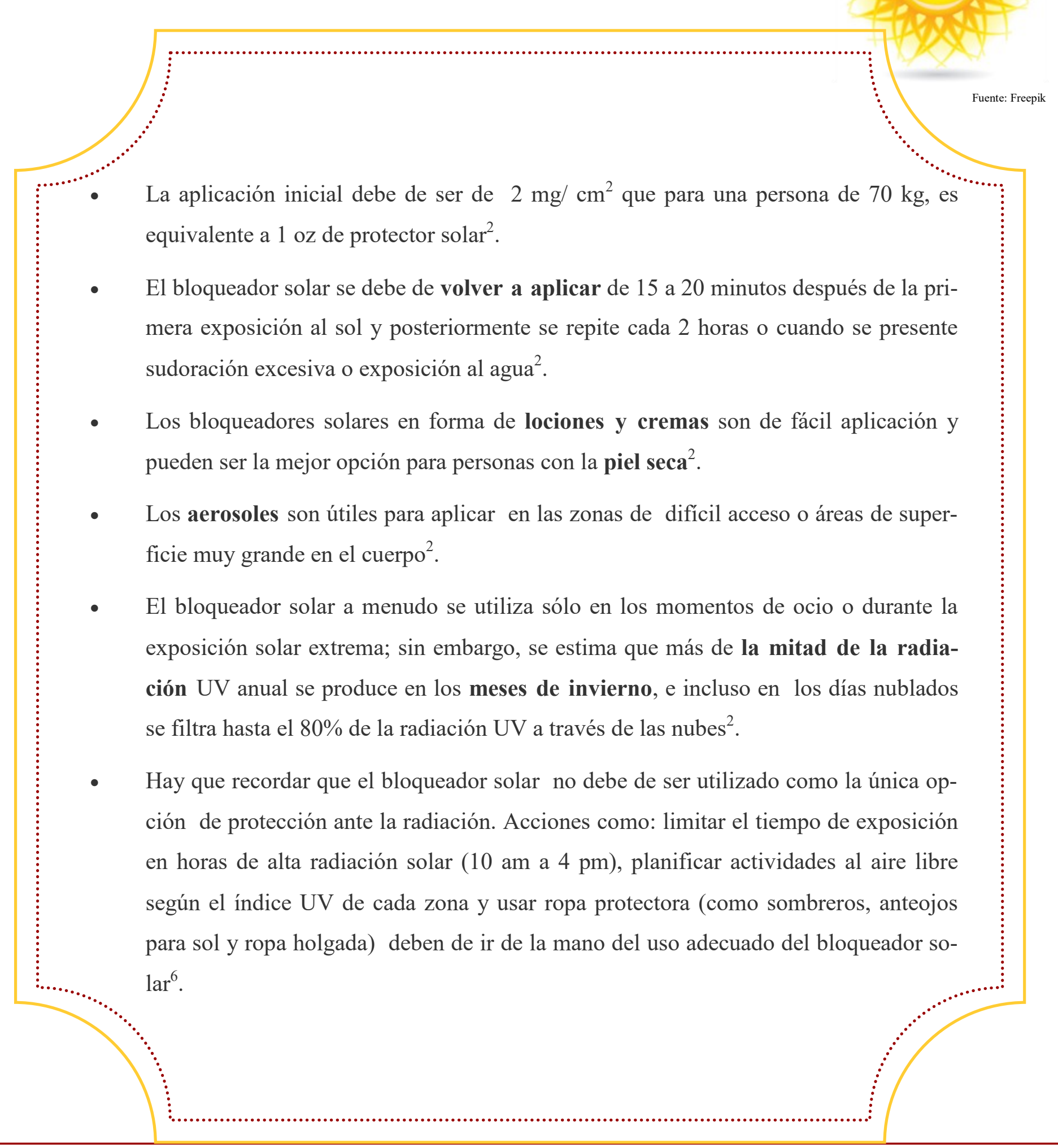




\section{Alertas de Farmacovigilancia}

- Riesgo de malformaciones congénitas y aborto espontáneo con micofenolato de mofetilo y micofenolato sódico (16/12/15): la Agencia Española de Medicamentos y Productos Sanitarios confirmó el potente efecto teratogénico del micofenolato de mofetilo y del micofenolato sódico en humanos. Si se administra en embarazo se incrementa el riesgo de malformaciones congénitas y de aborto espontáneo ${ }^{7}$.

Se estima la incidencia de malformaciones congénitas en un 23-27\% y se han reportado casos de aborto espontáneo en un $12-33 \%$ de las mujeres ${ }^{7}$.

Por lo tanto, se recomienda utilizar estos medicamentos en mujeres embarazadas sólo en caso de que no haya otra alternativa terapéutica disponible ${ }^{7}$.

Además, antes de administrar en mujeres en edad fértil, se debe confirmar la inexistencia de un embarazo y que la mujer esté utilizando medidas anticonceptivas efica$\operatorname{ces}^{7}$.

\section{Referencias}

1. Areias, C.; Almeida, D.; Mendes, C.; Kojima, M.; Sales de Oliveira , C.; Consiglieri, V.; Kaneko, T.; Rosado, C.; Mota, J.; Robles ,M.; Rolim, A. Functional photostability and cutaneous compatibility of bioactive UVA sun care products. Journal of Photochemistry and Photobiology B: Biology 148 (2015) :154-159.

2. Koshy, J.; Sharabi, S.; Jerkins, D.; Cox, J.; Cronin, S.; Hollier, L. Sunscreens: Evolving Aspects of Sun Protection.. Journal of Pediatric Health Care. (2010) 24, 343-346.

3. Schalka, S.; Silva, V. Sun protection factor: meaning and controversies. An Bras Dermatol. (2011): 86(3):507-15.

4. Garrote A. Protector Solar. Nuevos activos. Dermofarmacia. (2011). 30:3, 51-58.

5. Morabito, K.; Shapley, C.; Steeley, K.; Tripathi, A. Review of sunscreen and the emergence of non-conventional absorbers and their applications in ultraviolet protection. International Journal of Cosmetic Science. (2011) 33, 385-390.

6. WHO. Sun protection. A primary teaching resource. 2003. Disponible en: http://www.who.int/phe/uv. Consultado: 29/01/16.

7. Ministerio de Salud. Alerta de Seguridad 16-12-15. Disponible en: https://www.ministeriodesalud.go.cr/index.php/consulta-de-alertas-deseguridad/2849-16-de-diciembre-de-2015-alerta-de-seguridad-sobre-el-riesgo-de-malformaciones-congenitas-y-aborto-espontaneo-conmicofenolato-de-mofetilo-y-micofenolato-sodico/file. Consultado: 29/01/16. 